\title{
Generalization of Heart-Oriented Rhotrix Multiplication and its Algorithm Implementation
}

\author{
Ezugwu E. Absalom \\ Department of Mathematics \\ Ahmadu Bello University, \\ Zaria-Nigeria
}

\author{
Abiodun O. Ajibade \\ Department of Mathematics \\ Ahmadu Bello University, \\ Zaria-Nigeria
}

\author{
A. Mohammed \\ Department of Mathematics \\ Ahmadu Bello University, \\ Zaria-Nigeria
}

\begin{abstract}
This paper introduces a novel generalized concept of higher order heart-oriented rhotrix multiplication expression. The multiplication method we adapted is intended to maintain the unique properties associated with the initial definition of rhotrix. The special multiplication process can be used to solve various problems involving $n \quad x \quad n$ rhotrices multiplication. Most importantly a generalized expression for representing $\mathrm{n}$-dimensional rhotrix in a computational environment is presented. Sequential computational algorithm for higher order rhotrices multiplication is designed to buttress the mathematical expression, axioms and logic presented in this paper.
\end{abstract}

\section{General Terms}

Linear Algebra, Matrix theory, Algorithms

\section{Keywords}

Rhotrix heart, N-dimensional Rhotrix, Main Entries, Rhotrix Multiplication.

\section{INTRODUCTION}

Rhotrix is a new area of study relating to linear mathematical algebra. Rhotrix is defined as mathematical arrays which are, in some ways, between $(2 \times 2)$ and $(3 \times 3)$-dimensional matrices, and denoted matrix-tertions and noitrets [1]. Rhotrices multiplication was introduced and defined by [2], and an alternative method for multiplication of rhotrices as coupled matrices was given by [3]. This method was further given a generalization for $\mathrm{n}$-dimensional rhotrices in [4]. Their work was basically an idea that offers exercises for mathematical enrichment.

A set of rhotrices of dimension three was defined in [2] and extension in the dimension was considered possible. For instance a set of rhotrices of dimension five can be defined as<smiles>[R]C(C)(C)C(C)=C(C)CC(C)C</smiles>

where $h(R)$ is called the heart of $R$. The heart of a rhotrix is always defined as the element at the perpendicular intersection of the two diagonals of a rhotrix.

Rhotrix multiplication was previously defined in [2]. The scalar multiplication is defined as follows: let $\lambda \in \mathfrak{R}$ be a scalar and $\mathrm{R}_{\mathrm{n}}$ a rhotrix of dimension $n$, then

$$
R_{n}=\lambda\left(\begin{array}{ccccc} 
& & n & & \\
& o & p & q & \\
r & s & h\left(R_{n}\right) & t & u \\
& v & w & x & \\
& & y & &
\end{array}\right)=
$$

We consider, extending this definition to $\mathrm{n}$-dimensional rhotrix. Of interest in this paper for sake of illustration and clarity is five dimensional rhotrices given as follows:

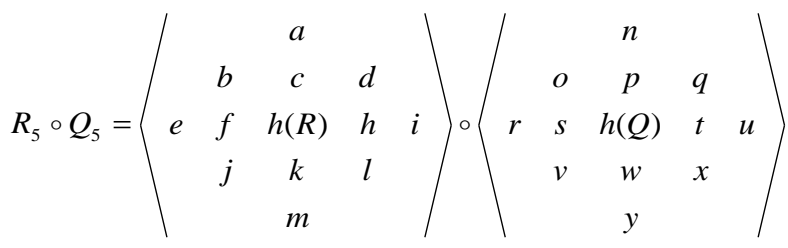

It should be noted that, the general concept of rhotrices is still at its elementary stage of development, as there are yet no established generalized form for its multiplication. Having ascertained this belief, the multiplication denoted by 'o', can be defined in many ways, we defined rhotrix multiplication based on the initial method given in [2] but with an extension to $\mathrm{n}$ dimension. The multiplication of the two rhotrices above is therefore given by; 
$=\langle e h(Q)+r h(R)$

Presented in this paper, is a generalized multiplication algorithm of $n$-dimensional rhotrices; which is an extension of work presented in [2]. The main objective of this work is to establish an ideal multiplicative concept and sequential computational algorithm suitable for higher dimensional heart-oriented rhotrix multiplication.

\section{RELATED WORK}

This section is almost a digression. It is devoted to the study of the simplest variant of the problem. In mathematics, the dot product, also known as the scalar product, is an operation which takes two vectors over the real numbers $R$ and returns a real-valued scalar quantity [5]. It is the standard inner product of the Euclidean space. In Euclidean geometry, the dot product, length, and angle are related: For a vector $a, a * a$ is the square of its length, and more generally, if $b$ is another vector

$$
a * b=|a| *|b| c \operatorname{so}(\theta)
$$

Where

- $|a|$ and $|b|$ denote the length (magnitude) of a and $b$,

- $\quad \theta$ is the angle between them.

Since $|a| \operatorname{cso}(\theta)$ is the scalar projection of $a$ onto $b$, the dot product can be understood geometrically as the product of the length of this projection and the length of $b$.

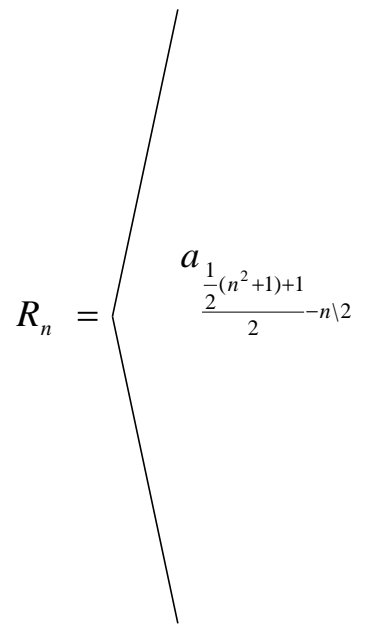

In linear algebra, the dot product of two vectors $a=\left[a_{1}\right.$, $\left.a_{2}, \ldots \ldots a_{n}\right]$ and $b=\left[b_{1}, b_{2}, \ldots . b_{n}\right]$ is defined as follows:

$a * b=\sum_{i=1}^{n} a_{i} * b_{i}=a_{1} * b_{1}+a_{2} * b_{2}+\ldots .+a_{n} * b_{n}$ where $\sum$ denotes summation notation.

The rhotrix main entries (vectors) or elements to heart multiplication could in some ways be likened to the same mathematical scalar dot product described above. In this paper, we aim to find an efficient implementation of this product computation on computers system. This implementation can also be considered an issue of prime importance when providing such systems with scientific software libraries or applications which use the dot product computation as data processing, especially for such area as rhotrices algebra which is a relatively new field of study.

\section{RESEARCH METHODOLOGY}

Ajibade indicated that the dimension of rhotrices can further be increased in size, but that uniquely a rhotrix would always have an odd dimension. He also indicated that a rhotrix $R_{n}$ of dimension $n$ will have $\left|R_{n}\right|$ entries where $\left|R_{n}\right|=\frac{1}{2}\left(n^{2}+1\right)$. Let's consider generalizing any given rhotrix $\left|R_{n}\right|$ with entries $a_{1}, a_{2} \ldots \ldots \ldots, a_{\frac{1}{2}\left(n^{2}+1\right)}$, then we have:

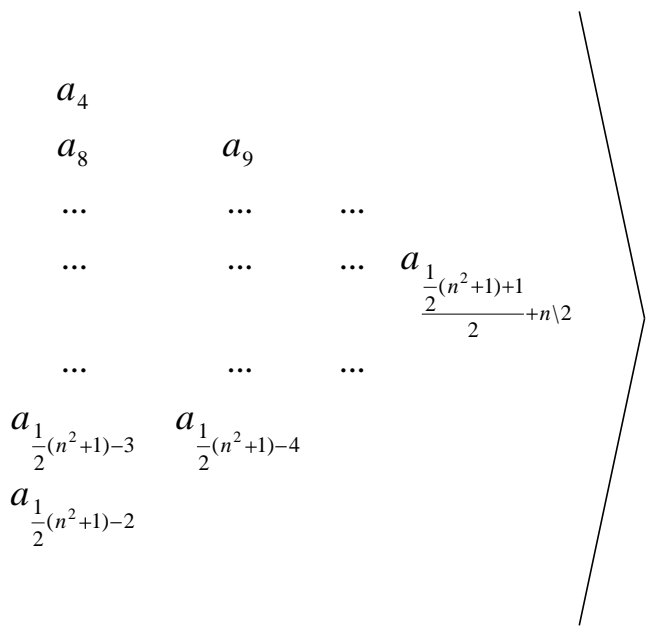




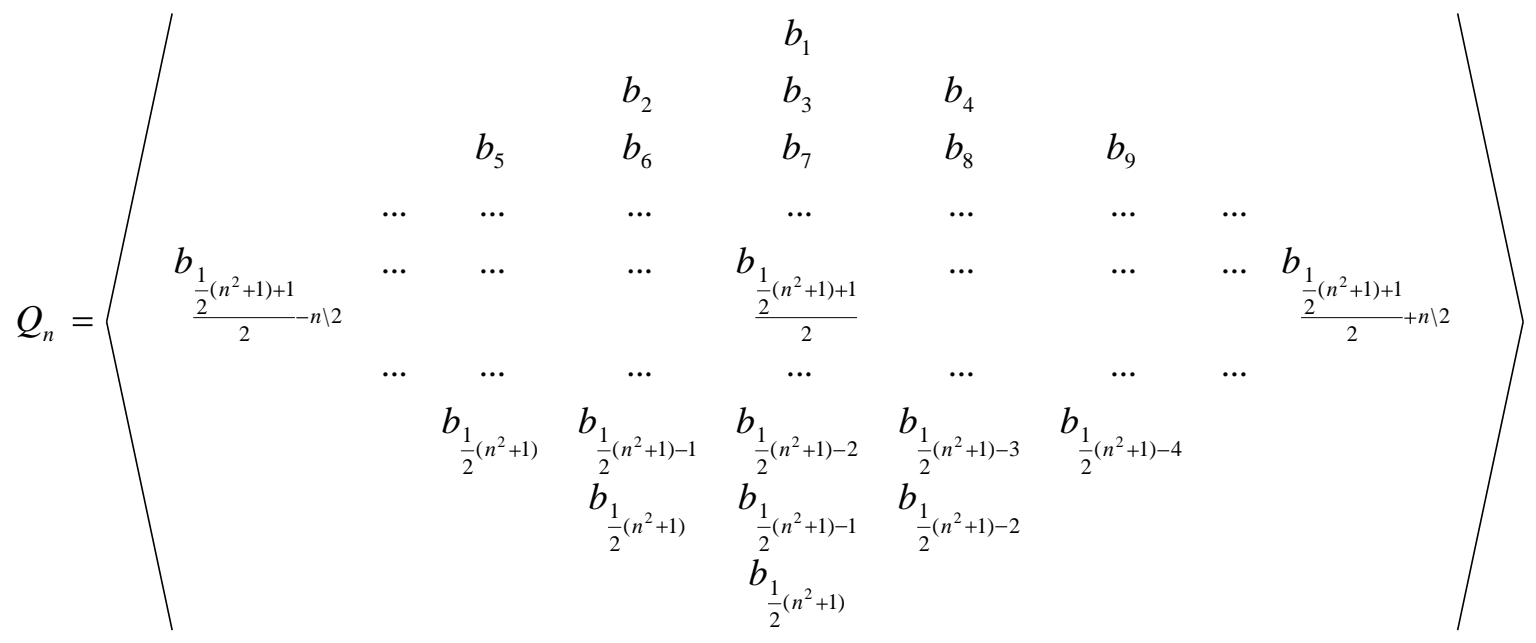

The symbol $\backslash$ in (1.4) denote integer division, in which case if $\mathrm{n}=5$, the $5 / 2$ gives 2 .

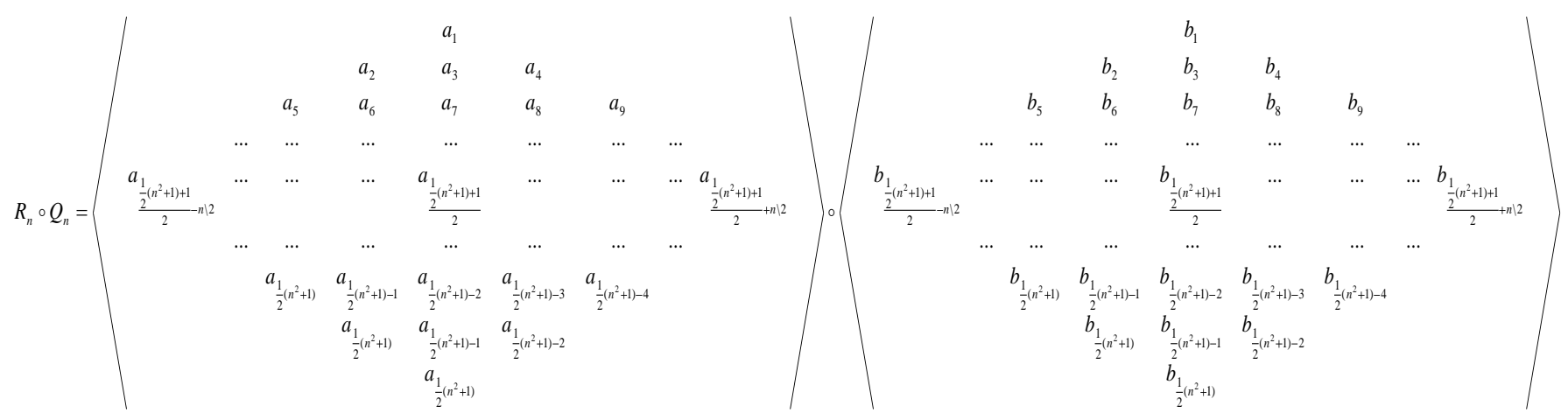

(1.5)
Similarly from (1.4), we can henceforth define the multiplication of any two heart-oriented rhotrices in the following way.

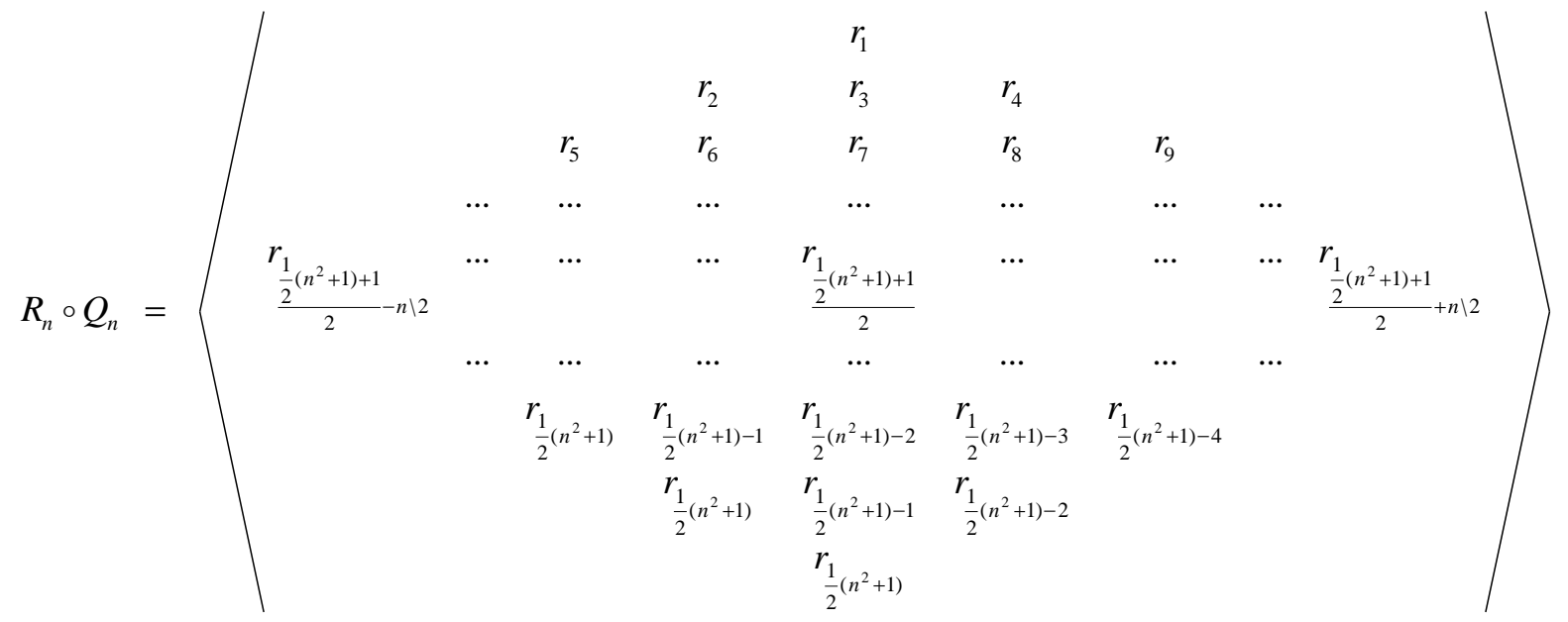

(1.6) 
Definition: (heart-oriented multiplication)

Let $a, b, c, \ldots \ldots, r \in \mathfrak{R}, \quad R_{n} \circ Q_{n}=a_{1} \times \frac{b_{\frac{1}{2}\left(n^{2}+1\right)+1}}{2}+$ $b_{2} \times a_{\frac{\frac{1}{2}\left(n^{2}+1\right)+1}{2}} \ldots a_{n} \times b_{\frac{1}{\frac{1}{2}\left(n^{2}+1\right)+1}}+b_{n} \times a_{\frac{\frac{1}{2}\left(n^{2}+1\right)+1}{2}}$.

Where $a_{\frac{\frac{1}{2}\left(n^{2}+1\right)+1}{2}}$ and $b_{\frac{1}{2}\left(n^{2}+1\right)+1}$ are the hearts of the two rhotrices

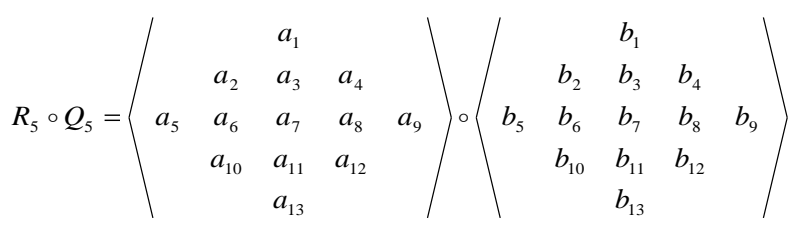

The multiplication of the rhotrices in (1.5) is commutative. The product function and operation is similar to that of the well known vector dot product, the only differences is that the resultant product for two rhotrices of (1.7) is a rhotrix of the same other as the two multiplicands shown in (1.8).

$$
\mathrm{R}_{5} \mathrm{OQ}_{5}=R=\left(\begin{array}{ccccc} 
& & r_{1} & \\
& r_{2} & r_{3} & r_{4} & \\
r_{5} & r_{6} & r_{7} & r_{8} & r_{9} \\
& r_{10} & r_{11} & r_{12} &
\end{array}\right)
$$

Rhotrix is usually characterized by its odd dimension, main entries (referred to as rhotrix vectors) and the hearts, which is the element at the perpendicular intersection of the two diagonals of a rhotrix. The heart is also very significant when dealing with rhotrix multiplication.

The multiplication process of the two rhotrices in (1.7) above can thus be expressed as:

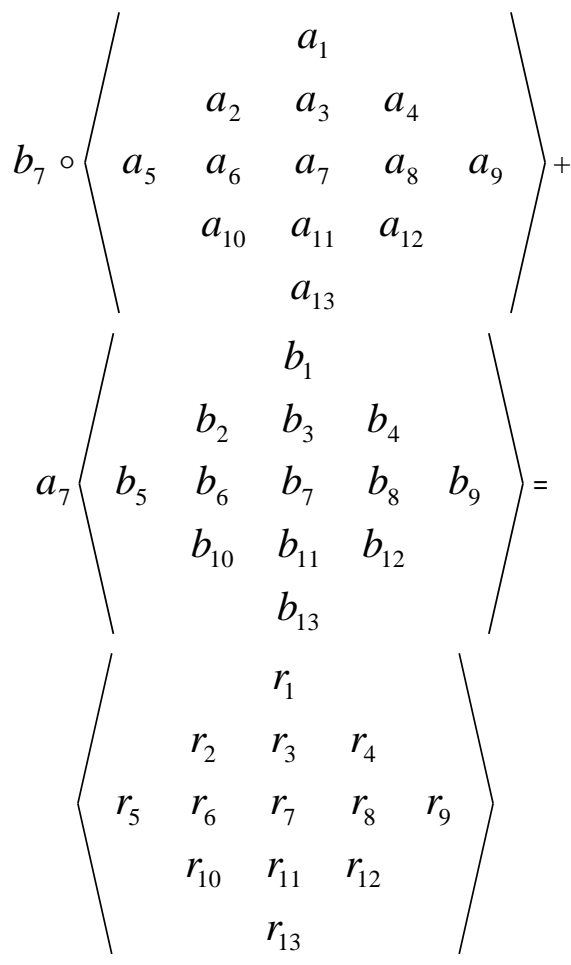

First, we need to know the values of the two rhotrices heart, $h(R)$ and $h(Q)$. Using our single-index notation to identify entries in rhotrix $\mathrm{R}$ and $\mathrm{Q}$, it turns out that the heart element $\mathrm{h}(\mathrm{R})$ and $\mathrm{h}(\mathrm{Q})$ can be identified by the index $\frac{\frac{1}{2}\left(n^{2}+1\right)+1}{2}$ , Where $\mathrm{n}$ is the rhotrix dimension. Hence, the multiplication is performed as follow.

$$
R_{5} \circ Q_{5}=R=\left(\begin{array}{ccccc}
\multicolumn{5}{c}{a_{1} b_{7}+b_{1} a_{7}} \\
& a_{2} b_{7}+b_{2} a_{7} & a_{3} b_{7}+b_{3} a_{7} & a_{4} b_{7}+b_{4} a_{7} & \\
a_{5} b_{7}+b_{5} a_{7} & a_{6} b_{7}+b_{6} a_{7} & a_{7} b_{7} & a_{8} b_{7}+b_{8} a_{7} & a_{9} b_{7}+b_{9} a_{7} \\
& a_{10} b_{7}+b_{10} a_{7} & a_{11} b_{7}+b_{11} a_{7} & a_{12} b_{7}+b_{12} a_{7} & \\
& & a_{13} b_{7}+b_{13} a_{7} &
\end{array}\right)
$$

$$
\begin{array}{lll}
r_{1}=a_{1} b_{7}+b_{1} a_{7} & r_{2}=a_{2} b_{7}+b_{2} a_{7} & r_{3}=a_{3} b_{7}+b_{3} a_{7} \\
r_{4}=a_{4} b_{7}+b_{4} a_{7} & r_{5}=a_{5} b_{7}+b_{5} a_{7} & r_{6}=a_{6} b_{7}+b_{6} a_{7} \\
r_{7}=a_{7} * b_{7} & r_{8}=a_{8} b_{7}+b_{8} a_{7} & r_{9}=a_{9} b_{7}+b_{9} a_{7} \\
r_{10}=a_{10} b_{7}+b_{10} a_{7} & r_{11}=a_{11} b_{7}+b_{11} a_{7} \\
r_{12}=a_{12} b_{7}+b_{12} a_{7} & r_{13}=a_{13} b_{7}+b_{13} a_{7}
\end{array}
$$

where $a_{7}$ and $b_{7}$ denote the hearts of the two rhotrices whose index is defined by $\frac{\frac{1}{2}\left(n^{2}+1\right)+1}{2}$, the resulting value of $\boldsymbol{r}_{7}$ is the product of the two hearts from the two rhotrices. We can now extend this to accommodate for rhotrix of n-dimension. Then going by the structure of (1.11), we can represent the rhotrix heart elements as $a_{\frac{\frac{1}{2}\left(n^{2}+1\right)+1}{2}}$ and $b_{\frac{\frac{1}{2}\left(n^{2}+1\right)+1}{2}}$, then, we have: 


$$
r_{n}=a_{n} * a_{\frac{\frac{1}{2}\left(n^{2}+1\right)+1}{2}}+b_{n} * a_{\frac{1}{2}\left(n^{2}+1\right)+1}^{2}
$$

By taking cumulative sum of the summation of $\boldsymbol{r}_{\boldsymbol{n}}$ from the principle of dot-product, equation (1.12) can be presented as follow:

$$
\sum_{n=1}^{\frac{1}{2}\left(n^{2}+1\right)} r_{n}=a_{n} * b_{\frac{\frac{1}{2}\left(n^{2}+1\right)+1}{2}}+b_{n} * a_{\frac{\frac{1}{2}\left(n^{2}+1\right)+1}{2}}
$$

Let us consider the multiplication of the following pair of rhotrices.

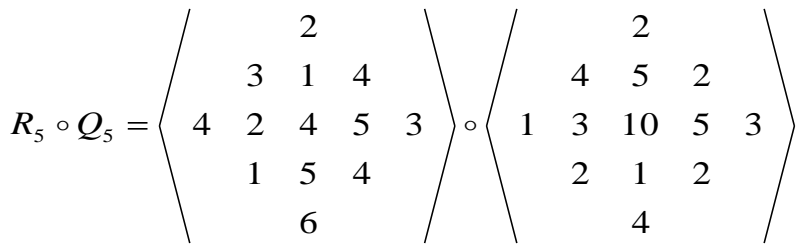

First we have to determine the hearts of the two rhotrices by applying the formula $h\left(R_{n}\right)=\frac{\frac{1}{2}\left(n^{2}+1\right)+1}{2}$ and $h\left(Q_{n}\right)=\frac{\frac{1}{2}\left(n^{2}+1\right)+1}{2}$. The dimension $\boldsymbol{n}$ of the two rhotrices is given to be 5 , therefore the hearts of $h\left(R_{5}\right)$ and $h$ $\left(\mathrm{Q}_{5}\right)$ on substitution is the $3^{\text {rd }}$ element eitsher from top to bottom or left to right along the intersection of the rhotrix diagonals. The value of $a_{7}$ and $b_{7}$ are 4 and 10 respectively. On multiplication, we then have:

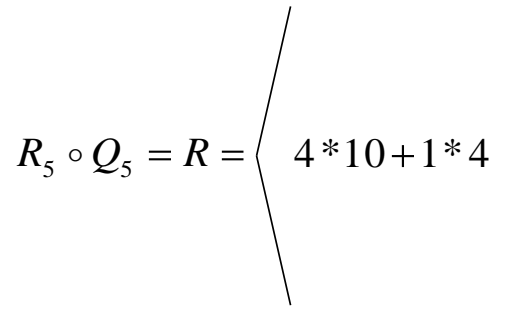

Substituting this computation into (1.12), we then have:

$$
\begin{array}{ll}
R_{1}=2 * 10+2 * 4 & R_{2}=3 * 10+4 * 4 \\
R_{3}=1 * 10+5 * 4 & R_{4}=4 * 10+2 * 4 \\
R_{5}=4 * 10+1 * 4 & R_{6}=2 * 10+3 * 4 \\
R_{7}=4 * 10 & R_{8}=5 * 10+5 * 4 \\
R_{9}=3 * 10+3 * 4 & R_{10}=1 * 10+2 * 4 \\
R_{11}=5 * 10+1 * 4 & R_{12}=4 * 10+2 * 4 \\
R_{13}=6 * 10+4 * 4 &
\end{array}
$$$$
R_{5} \circ Q_{5}=R=\left(\begin{array}{rrrrr} 
& & 28 & & \\
& 46 & 30 & 48 & \\
44 & 32 & 40 & 70 & 42 \\
& 18 & 54 & 48 & \\
& & 76 & &
\end{array}\right)
$$

The obtained result differs with that of B. Sani's row-column method of rhotrices multiplication [3][4]; this might be, of course, due to the simple facts that the approach given in [2] preferred to identify rhotrices as a unique mathematical algebraic objects different from the traditional matrix algebra.

$$
2 * 10+2 * 4
$$$$
1 * 10+5 * 4
$$$$
4 * 10
$$$$
4 * 10+2 * 4
$$$$
5 * 10+5 * 4 \quad 3 * 10+3 * 4
$$$$
5 * 10+1 * 4
$$$$
4 * 10+2 * 4
$$$$
6 * 10+4 * 4
$$

\section{RESULT AND DISCUSSIONS}

This section presents a computational sequential algorithm for $\mathrm{n} \times \mathrm{n}$ rhotrix multiplication, the implementation of the algorithm was first written in $\mathrm{C}$ programming language and tested on Intel uni-processor system. The hearts-oriented multiplication algorithm is as shown in figure 1:

Algorithm: Rhotrix multiplication (heart oriented):

Input:

a[0.. $\left.\frac{1}{2}\left(n^{2}+1\right)\right]$;

$b\left[0 . . \frac{1}{2}\left(n^{2}+1\right)\right]$;

Output:

$$
\begin{aligned}
& r\left[0 . . \frac{1}{2}\left(n^{2}+1\right)\right] ; \\
& \text { for } i=: 1 \text { to } \frac{1}{2}\left(n^{2}+1\right) \\
& \quad i f\left(i==\frac{\frac{1}{2}\left(n^{2}+1\right)+1}{2}\right) \\
& r[i] \leftarrow a\left[\frac{\frac{1}{2}\left(n^{2}+1\right)+1}{2}\right] * b\left[\frac{\frac{1}{2}\left(n^{2}+1\right)+1}{2}\right] ;
\end{aligned}
$$

else 
$r[i] \leftarrow a[i] * b\left[\frac{\frac{1}{2}\left(n^{2}+1\right)+1}{2}\right] * b[i] * a\left[\frac{\frac{1}{2}\left(n^{2}+1\right)+1}{2}\right]$

endif

endfor

Figure1. Recursive, heart-oriented rhotrix multiplication algorithm

The snapshot of computer generated result of our executed program is shown below:

The First Rhotrix R (A) [0] Is:

$$
\begin{array}{ll}
R(A)[1]= & 2 \\
R(A) ~[2]= & 3 \\
R(A) ~[3]= & 1 \\
R(A) ~[4]= & 4 \\
R(A) ~[5]= & 4 \\
R(A) ~[6]= & 2 \\
R(A) ~[7]= & 4 \\
R(A) ~[8]= & 5 \\
R(A) ~[9]= & 3 \\
R(A) ~[10]= & 1 \\
R(A) ~[11]= & 5 \\
R(A) ~[12]= & 4 \\
R(A) ~[13]= & 6
\end{array}
$$

The Second Rhotrix R (B) [0] Is:

$$
\begin{array}{ll}
\mathbf{R}(B)[1]= & 2 \\
\mathbf{R}(B)[2]= & 4 \\
\mathbf{R}(B)[3]= & 5 \\
R(B)[4]= & 2 \\
R(B)[5]= & 1 \\
R(B)[6]= & 3 \\
R(B)[7]= & 10 \\
R(B)[8]= & 5 \\
R(B)[9]= & 3
\end{array}
$$

$$
\begin{array}{ll}
R(B)[10]= & 2 \\
R(B)[11]= & 1 \\
R(B)[12]= & 2 \\
R(B)[13]= & 4
\end{array}
$$

Linear multiplication of the above two rhotrices are:

$$
\begin{aligned}
& R[1]=28 \\
& R[2]=46 \\
& R[3]=30 \\
& R[4]=48 \\
& R[5]=44 \\
& R[6]=\quad 32 \\
& R[7]=40 \\
& R[8]=\quad 70 \\
& R[9]=42 \\
& R[10]=\quad 18 \\
& R[11]=\quad 54 \\
& R[12]=48 \\
& R[13]=\quad 76
\end{aligned}
$$

Formatted multiplication of the above two rhotrices are:

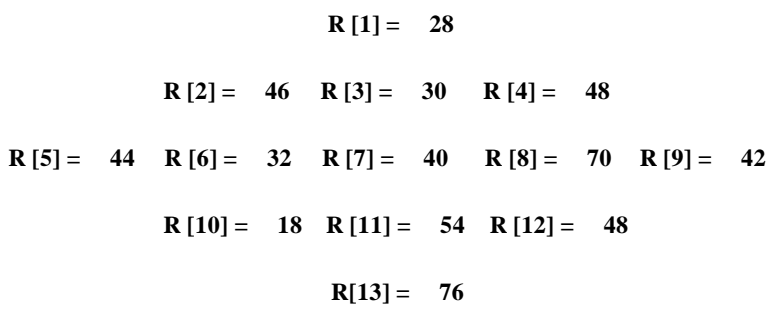

It was observed that the theoretical result of the multiplication correspond with the program generated result, hence this prove the correctness of our algorithm. The experimental result shows that we can represent rhotrix multiplication on a computer system independent of the shape and dimension of the given rhotrix. The algorithm used in the experimental process is scalable and can be extended to cover any size of rhotrix, which is always of odd dimension.

\section{CONCLUSION}

This paper has presented an extended multiplication method, derivation and algorithm for higher order rhotrices multiplication. The method presented is quite unique and directed only to rhotrix multiplication of the form discussed in [2]. In particular we have shown that a generalized equation 
as in our case could be used to implement n-dimensional rhotrices multiplication as against the row-column multiplication method presented in [3] [4].

\section{ACKNOWLEDGMENTS}

We thank Junaidu B. Sahalu, Ahmadu Bello University for his numerous comments and suggestions, which greatly improved the final version of the paper.

\section{REFERENCES}

[1] Atanassov. K.T. and Shannon, A.G., 1998, International Journal of Mathematics Education in Science and Technology, 29, 898-903.
[2] Ajibade, A. O., 2003, the concept of rhotrix in mathematical enrichment, International Journal of Mathematical Education in Science and Technology, Vol. 34:2s, 175-179.

[3] Sani, B. 2004, the row-column multiplication of high dimensional rhotrices, International Journal of Mathematical Education in Science and Technology, Vol.35:5, 777-781.

[4] Sani, B. 2007, Conversion of a rhotrix to a 'coupled matrix', International Journal of Mathematical Education in Science and Technology, Vol. 38:5, 657-662.

[5] Singh S. K. "Scalar (dot) product", http://cnx.org/content/m14513/1.5/, (Accessed Nov. 2010). 